\title{
A Continuum Model for Simulating Crowd Turbulence
}

\author{
Abhinav Golas ${ }^{1} \quad$ Rahul Narain $^{2} \quad$ Ming Lin ${ }^{1}$ \\ ${ }^{1}$ University of North Carolina at Chapel Hill, $\quad{ }^{2}$ University of California, Berkeley
}

\section{Introduction}

With increasing world population, we are observing denser and denser crowds in public places. This has led to an increasing incidence of crowd disasters at high densities, known collectively as crowd turbulence [Helbing et al. 2007]. There is an urgent need to understand and simulate such crowds in order to facilitate emergency response, as well as prediction and planning to prevent such emergencies. Simulated crowd turbulence can also be used to augment the fidelity of virtual environments in computer games and movies. In addition, for real-time prediction and response, interactive simulation is an essential requirement.

However, crowds in such scenarios demonstrate behavior that deviates significantly from well-known crowd patterns and cannot be simulated using existing algorithms. In fact, as noted by Helbing et al.[2007], traditional regimes of modeling crowd navigation cannot be used to simulate crowd turbulence.

We use two essential insights to propose a new physically-based crowd model capable of replicating crowd turbulence. The first is an acceleration model for agents that is cognizant of the finite acceleration capabilities of humans, as well as the implied inertia. The second is a model for inter-personal contact stress, incorporating normal pressure forces, and tangential frictional forces that agents exert on contact at high densities.

\section{Crowd Turbulence}

Under normal conditions, crowds of pedestrians move smoothly with slowdowns and fluctuations only near obstacles. However, the behavior of crowds changes dramatically as the density and urgency of pedestrians increase. First, the crowd begins to exhibit stopand-go waves akin to vehicular traffic. The motion of pedestrians alternates between stopped high-density regions, and those moving forward at low density, with these density waves moving backwards through the crowd. Second, at even higher densities, as pedestrians panic, the crowd shows chaotic oscillatory behavior reminiscent of turbulence in fluids, with pedestrians being pushed in all directions even backwards - against their desired direction of motion. The term "crowd turbulence" is used to desribe crowds in such conditions.

We find that stop-and-go waves arise naturally in simulated crowds that observe speed sensitivity to density and limited acceleration akin to those demonstrated by humans. As has been noted in observations of turbulent crowds, their behavior bears significant similarities to granular materials. Our proposed model for inter-personal contact stress follows this insight to simulate crowd turbulence.

\section{Stress Modeling for Crowds}

We propose that behavior of crowds at high densities is guided by the desire to reach regions of lower densities, i.e. to reduce discomfort caused by high densities. To do so, agents move along the gradient of a discomfort function $g(\rho)$, where $\rho$ is the crowd density. However, reduction of discomfort alone is insufficient for replicating crowd turbulence. That relies on an accurate model for inter-personal friction. Crowds can exert either static friction where there is no relative motion between agents, or kinetic friction when agents have relative shear motion. This model can be recast to the models for granular materials, using which stress can be computed interactively.

This model is coupled with an existing crowd navigation model of

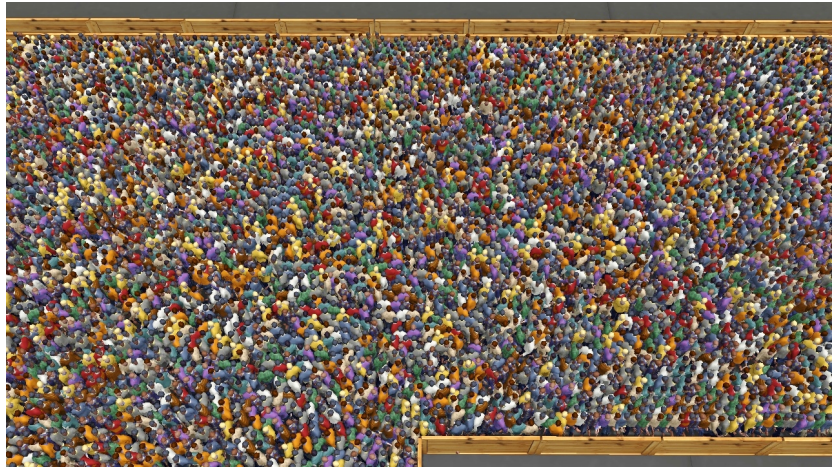

(a)

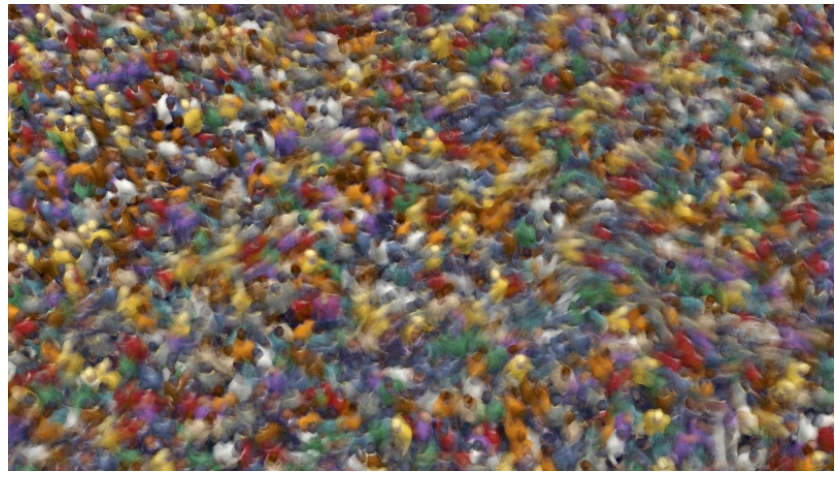

(b)

Figure 1: (a) A screenshot of the Hajj simulation, where two merging groups of agents head east (b) A time-averaged inset showing oscillations caused by simulated crowd turbulence

[Golas et al. 2013] for low-medium densities. Since the discomfort function $g(\rho)$ is constant below a critical density, crowd behavior remains unchanged at low densities. At higher densities, increasing discomfort starts to affect crowd behavior, its contribution rising with increasing density.

\section{Results}

We have validated our model against known instances of crowd turbulence, notably the Hajj [Helbing et al. 2007], and the Love Parade disaster of 2010. Fig. 1 shows a screenshot of simulated agents in Hajj scenario, where two groups of agents from the west and south merge while heading into a constrained corridor heading east. The time averaged inset shows oscillation in directions orthogonal to the desired direction of motion - one of the clear indicators of crowd turbulence. This oscillation has a certain structure, as shown by stationary and oscillating clusters of agents. For more detailed analysis, please refer to the supplementary video.

\section{References}

Golas, A., Narain, R., Curtis, S., And Lin, M. C. 2013. Hybrid long-range collision avoidance for crowd simulation. IEEE Transactions on Visualization and Computer Graphics 99, PrePrints, 1.

Helbing, D., Johansson, A., And Al-Abideen, H. Z. 2007. Dynamics of crowd disasters: An empirical study. Phys. Rev. E 75 (Apr), 046109. 